\title{
Peripheral venous pressure to monitor fluid resuscitation in burns-more confusing than helpful?
}

\section{Lavrentieva Athina}

Optimizing cardiovascular function to ensure adequate tissue oxygen delivery is a key objective in the care of critically ill burn patients. Pronounced hemodynamic fluctuation during initial burn resuscitation, the Intensive Care Unit stay, or during major surgical procedures requires close monitoring of hemodynamic status. The choice of which hemodynamic monitor to use on critically ill burn patients depends on the physician's knowledge of the basic technical principles of the device's function; convenient and effective device use; device safety and accuracy, and reliability compared to the current standard of care. ${ }^{[1]}$ The effectiveness of hemodynamic monitoring depends both on available technology and on our ability to diagnose and effectively treat the disease for which it is used.

The present study evaluated the use of peripheral venous pressure (PVP) monitoring and demonstrated reliable agreement between central venous pressure (CVP) and PVP over a $10 \mathrm{~h}$ period, suggesting that $\mathrm{PVP}$ monitoring can be used as a simple, cost-effective, and less invasive substitute for CVP monitoring in patients admitted to the burns Intensive Care Unit. ${ }^{[2]}$ This method may have potential implications for clinical situations where CVP is used for patient care decisions, in situations where central venous site is inaccessible and also to avoid the complications of central venous catheterization in critically ill burns patients.

The question that however arises is whether the measurement of CVP and PVP, as its surrogate, has clinical utility and whether it will be able to help us to assess the hemodynamic status of our patients and change our therapeutic approach in order to improve their outcome. Recent clinical review ${ }^{[3]}$ emphasizes that $^{2}$

From:

Intensive Care Unit, Papanikolaou Hospital, Thessaloniki, Greece

Correspondence:

Dr. Lavrentieva Athina, Papanikolaou Hospital, Thessaloniki, Greece.

E-mail: alavrenti@gmail.com

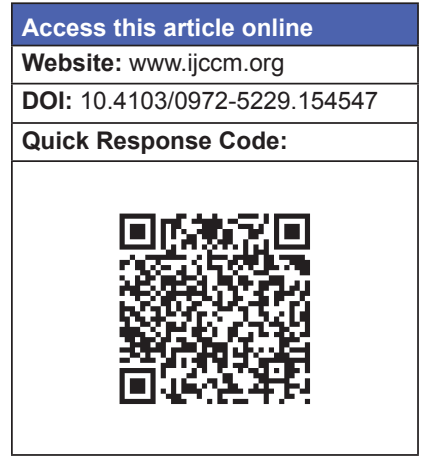

hemodynamic monitoring can only improve outcomes if three conditions are met: The data obtained from the monitoring device must be sufficiently accurate to be able to influence therapeutic decision making; the data obtained from the monitoring system must be relevant to the patient being monitored; and changes in management made as a result of the data obtained need to be able to improve outcomes. If these three conditions are not met, monitoring is unlikely to be associated with improved clinical outcome in critically ill patients regardless of the monitoring technique.

Many different monitoring systems with different degrees of invasiveness are now available, and physicians may feel somewhat confused by the range of options. Classifying them according to how accurate (closeness of measured values to the "true" value, expressed as the bias) or precise (variability of values due to random errors of measurement) they are is difficult because of the lack of a perfect "gold" standard for comparison. ${ }^{[3]}$ The present study evaluated the usefulness of PVP measurement by comparing monitoring data with data obtained from the use of central venous catheter as the reference; PVP trends seem to provide equivalent physiological information to CVP trends in this specific subset of patients. As changes in PVP parallel changes in CVP, the authors concluded that PVP monitoring may offer an alternative to direct CVP measurement for 
estimation of volume status and for guiding fluid therapy in burn patients.

However, a word of caution is needed here. The basis for using CVP and as its surrogate, PVP to guide fluid management comes from the belief that CVP reflects intravascular volume; specifically, it is widely believed that patients with low CVP are volume depleted, while patients with high CVP are volume overloaded. However, data suggest that this approach may be questionable, and the monitoring of CVP to guide fluid therapy has its own major limitations. ${ }^{[4]}$

Central venous pressure is considered to be the back-pressure to systemic venous return. ${ }^{[1]}$ Elevation in CVP reflects right ventricular pressure overload, although this gives no information on the precise etiology involved. CVP seems to be a measure of right atrial pressure alone; and not a measure of blood volume or ventricular preload. Recent publications referred to a very weak relationship between CVP and blood volume, as well as the inability of CVP to predict the hemodynamic response to fluid challenge. ${ }^{[5,6]}$ It has been recognized that measurements of the "static" cardiac filling pressure cannot accurately reflect preload or fluid responsiveness. It may be more useful to monitor "dynamic" parameters, for example, pulse pressure variation, to detect hypovolemia and guide fluid therapy in critically ill patients ${ }^{[3]}$ On the other hand, measurement of CVP may be useful in select circumstances, such as in patients who have undergone a heart transplant, or in those who have suffered a right ventricular infarction or acute pulmonary embolism. ${ }^{[4]}$ In these cases, CVP may be used as a marker of right ventricular function rather than an indicator of volume status.

The present study ${ }^{[2]}$ describes a simple, inexpensive, and minimally invasive technique that can be used as a substitute to CVP and demonstrates the acceptable accuracy of PVP monitoring (the changes in CVP and PVP are strongly correlated and consistent over time). Considering that the clinical utility of CVP as a guide to diagnosis or therapy in critically ill patients continuous to be disputed; also considering the fact that PVP provides equivalent physiological information to $C V P,{ }^{[7,8]}$ the clinical usefulness of PVP monitoring is questionable. An isolated measurement of CVP or PVP, like any other single hemodynamic variable, cannot describe the state of the circulation. However, when history and physical examination are insufficient, clinicians can integrate CVP into hemodynamic assessment along with other monitoring such as a functional hemodynamics and echocardiography. ${ }^{[9]}$

Of the three aforementioned conditions required for outcome benefit with the use of monitoring systems, only the first seems to be fulfilled. Although this study assessed the agreement of CVP with PVP and demonstrated such an agreement, no definite conclusion can be drawn about the clinical usefulness of PVP monitoring in terms of fluid management in burn patients.

\section{References}

1. Pinsky MR, Payen D. Functional hemodynamic monitoring. Crit Care 2005;9:566-72.

2. Sherif L, Joshi VS, Ollapally A, Jain P, Shetty K, Ribeiro KS et.al. Peripheral venous pressure as a reliable predictor for monitoring central venous pressure in patients with burns. Ind. J Crit Care Med. 2015; 19:199-202.

3. Vincent JL, Rhodes A, Perel A, Martin GS, Della Rocca G, Vallet B, et al. Clinical review: Update on hemodynamic monitoring - A consensus of 16. Crit Care 2011;15:229.

4. Marik PE, Baram M, Vahid B. Does central venous pressure predict fluid responsiveness? A systematic review of the literature and the tale of seven mares. Chest 2008;134:172-8.

5. Pinsky MR, Kellum JA, Bellomo R. Central venous pressure is a stopping rule, not a target of fluid resuscitation. Crit Care Resusc 2014;16:245-6.

6. Cecconi M, De Backer D, Antonelli M, Beale R, Bakker J, Hofer C, et al. Consensus on circulatory shock and hemodynamic monitoring. Task force of the European Society of Intensive Care Medicine. Intensive Care Med 2014;40:1795-815.

7. Kim SH, Park SY, Cui J, Lee JH, Cho SH, Chae WS, et al. Peripheral venous pressure as an alternative to central venous pressure in patients undergoing laparoscopic colorectal surgery. Br J Anaesth 2011;106:305-11.

8. Stoneking L, Deluca LA Jr, Fiorello AB, Munzer B, Baker N, Denninghoff KR. Alternative methods to central venous pressure for assessing volume status in critically ill patients. J Emerg Nurs 2014;40:115-23.

9. Berlin DA, Bakker J. Starling curves and central venous pressure. Crit Care 2015;19:776.

How to cite this article: Athina L. Peripheral venous pressure to monitor fluid resuscitation in burns-more confusing than helpful?. Indian J Crit Care Med 2015;19:197-8.

Source of Support: Nil, Conflict of Interest: None declared. 